\title{
IOT BASED VITAL SIGNS MONITORING SYSTEM FOR HUMAN BEINGS
}

Kavipriya Sundaravadivel

UG Student, Department of Biomedical Engineering, Sethu Institute of technology, Kariapatti, Virudhunagar-626115, Tamilnadu, (India).

E-mail: kavipriya882002@gmail.com

ORCID: https://orcid.org/0000-0002-4215-3318

Priyadharshini Malaiyarasan

UG Student, Department of Biomedical Engineering, Sethu Institute of technology, Kariapatti, Virudhunagar-626115, Tamilnadu, (India).

E-mail: dharshinimalaiyarasan@gmail.com

ORCID: https:// orcid.org/0000-0002-7050-0663

Hemalatha Karuppiah

Professor, Department of Biomedical Engineering, Sethu Institute of technology, Kariapatti, Virudhunagar-626115, Tamilnadu, (India).

E-mail: hemaa75@gmail.com

ORCID: https://orcid.org/0000-0002-4356-9905

Nageshwari Raja

Professor, Department of Biomedical Engineering, Sethu Institute of technology, Kariapatti, Virudhunagar-626115, Tamilnadu, (India).

E-mail: nageshwari.raja@gmail.com

ORCID: https://orcid.org/0000-0001-9717-3714

Recepción: 29/11/2019 Aceptación: 02/09/2020 Publicación: 30/11/2021

\section{Gitación sugerida:}

Sundaravadivel, K., Malaiyarasan, P., Karuppiah, H., y Raja, N. (2021). IoT based vital signs monitoring system for human beings. 3C Tecnología. Glosas de innovación aplicadas a la pyme, Edición Especial, (noviembre, 2021), 611-621. https://doi.org/10.17993/3ctecno.2021.specialissue8.611-621 


\section{ABSTRACT}

Evolution of human lifestyle paves the way for number of chronic health issues such as diabetes, cardiovascular diseases and others in worldwide. Scientific community devices modern technology devices to proactive health management. The internet of things (IoT) is an emerging technology that is expected to propagate in all aspects of our day-to-day life. Vital signs are essential indications for diagnosing and treating life threatening disease in an early stage. It includes body temperature, muscular activity, pulse rate, blood pressure and respiratory rate. Routine measurement of these physical signs witnesses the healthy human status and to certify health of the human. The aim of the present study was to develop IoT based vital signs monitoring system to assess, improve and assist the health of human beings who are hospitalized or at home in a real-time manner. IoT based healthcare monitoring system displays these measured data in mobile application using internet connectivity. This system helps both the physician and patient to access the healthcare related information anytime and anywhere in a real time manner and mainly consist of sensors namely, DS18B20 temperature sensor, pulse sensor, and EMG V3 sensor and node MCU which is used as a microcontroller. The patient's temperature, pulse rate and muscular activity are assessed, displayed and stored by the systems and sent to the physician mobile phone directly. Thus, this IOT based vital signs monitoring system effectively provide an accurate detection of disease in an early manner and monitor the patient's health status continuously and remotely.

\section{KEYWORDS}

Health monitoring, IoT, Vital parameters, Visualization. 


\section{INTRODUCTION}

Now-a-days, vital signs monitoring plays a major role in healthcare. Vital signs are the basic indicators of the human's health status. Continuous vital signs monitoring becomes inexpensive by wide ranges wearable sensors. There are number of reasons for measuring vital signs which provides information like (1) existence of an acute medical problems. (2) Quantifying the illness of the body occurred due to physiological stress (3) can be used as a diagnostic marker for chronic diseases such as hypertension, diabetes and cardiovascular diseases (Castledine, 2006).

Vital signs are an important element for deducing the progress of the treatment especially in heart diseases and also for determining the type of treatment protocol to be followed for treating the patient. To address the quality health status, the number of prototypes and commercial products has been used in healthcare monitoring system that has the advantage of managing and monitoring chronic diseases of elderly people and post-operative patients (Pantelopoulos \& Bourbakis, 2010).

Normally vital signs changes with age, sex, height and state of physical activity. Body temperature is the measure of the body's sensitivity to heat or cold. The normal body temperature of an individual is $98.6 \mathrm{~F}$. Pulse rate is the measure of number of heart beats for one minute which is normally measured in bpm (beats per minute).

The normal heartbeat about 60 to 80 times a minute. Blood pressure is the force of blood against the arterial wall. The normal blood pressure of the human is 120/80 $\mathrm{mmHg}$. Breathing is the process of movement of air in and out of the lungs. For a healthy human, the typical respiratory rate at the rest would be 12-18 breaths per minute.

Muscular activity accounts for much of a body's energy consumption. Normally muscular activity is measured in the range of $50 \mu \mathrm{V}$ to $30 \mathrm{mV}$ using EMG (Hong et al., 2011). Vital signs are an important element for deducing the progress of the treatment especially in heart diseases and for determining the type of treatment protocol to be followed for treating the patient.

Long term recording and management of vital signs parameters helps the doctors to understand much better prognosis of health (Malhi et al., 2012). Long term recording 
and management of vital signs parameters helps the doctors to understand much better prognosis of health (Hassanalieragh et al., 2015). Increased use of mobile devices that has major advantages of recent technologies add benefit to human health. Acquiring higher standard of health is a fundamental right of an individual (Senthamilarasi et al., 2018).

Thus, our design provides conventional clinical setting that decrease cost of maintain health. The present work focuses on the design and development of modern healthcare system utilizing IoT technology to monitor vital physiological parameters.

\section{MATERIALS AND METHODS}

\subsection{OVERVIEW}

The main goal of the project is to measure the vital signs parameters whenever and wherever necessary. Here three basic vital signs such as temperature, muscle activity and pulse rate are measured by DS18B20 sensor, EMG v3 sensor and pulse sensor respectively.

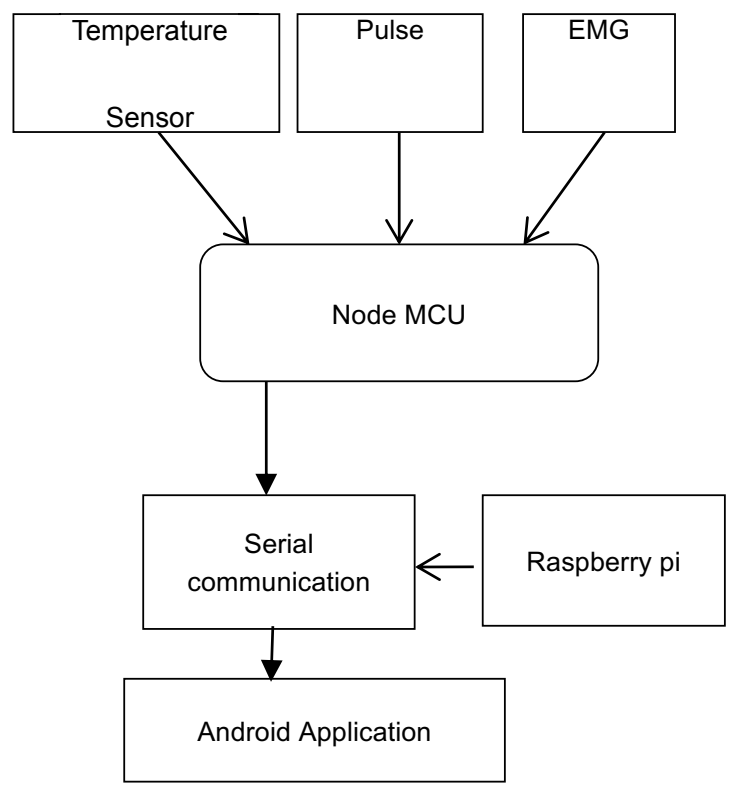

Figure 1. Block diagram of loT based vital signs monitoring system.

Source: own elaboration. 


\subsection{NODE MCU}

Node MCU is a firmware on ESP8266. It is basically a SoC (System on Chip). Node MGU includes a self-contained Wi-Fi networking solution offering as a bridge from existing microcontroller to Wi-Fi and also capable of running an application. Operating voltage is 3.3 volt. Node MCU is Wi-Fi trans-receiver chip. The baud rate of the Node MCU is the number of symbols transmitted per second. The baud rate is 115200 . The node MCU has 4 MB internal flash memory. The stored data in the ESP8266 can be accessed to the internet through local Wi-Fi connection using proper user ID and password.

\subsection{TEMPERATURE SENSOR}

The temperature sensor DS18B20 is a digital thermometer and provides 9-12 bit temperature reading. Information is sent to / from DS18B20 over 1-wire interface hence only one wire (and ground) needs to be connected from a central microprocessor to DS18B20. It can be powered from data line. Power supply range is 3-5.5 volt.

\subsection{PULSE SENSOR}

The pulse sensor is a heart rate monitoring sensor and is easily programmed by Arduino IDE software. Pulse sensor obtains the heart rate by non- invasive monitoring and has inbuilt noise cancellation circuit for better output. The operating voltage is 3.3 volt.

\subsection{EMG SENSOR}

EMG sensor measures the electrical activity of the muscles at rest and during contraction. Any movement in the muscular cells causes electrical signals. This signal propagates through adjacent tissues and bones. Signals are incorporated through sensors in microvolt. Three electrodes are fixed to the surface of the body for measuring EMG. Sensor is connected to $9 \mathrm{~V}$ batteries. The EMG sensor is also powered by external batteries.

\subsection{RASPBERRY PI}

Raspberry $\mathrm{Pi}$ is a single board computer that runs Linux and is powered using USB by connecting it to the PG. The SD card is inserted to the Raspberry Pi which is used as memory unit for loading the operating system. The Raspberry Pi doesn't have any memory 
source. It also provides a set of GPIO pins that allows controlling electronic components for exploring IoT and operating voltage is $5 \mathrm{~V}$.

\subsection{IOT}

IoT extends the internet connectivity to physical objects and the data transfer is made between the connected devices. It can transfer the data without human to human or human to computer interaction. The IoT is the real time data transfer system through gateway.

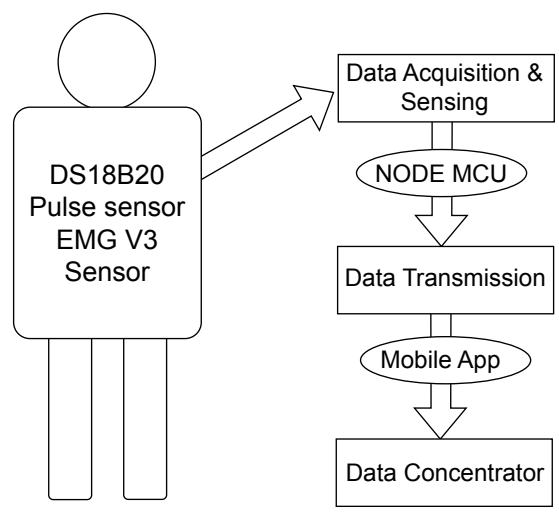

Figure 2. Architecture of IOT based vital signs monitoring system.

Source: own elaboration.

Data Acquisition is performed by multiple sensors (DS18B20 temperature sensor, pulse sensor and EMG V3 sensor) which measure the physiological changes of the body. The sensors are connected to the network through data aggregators which is typically a mobile app used by the subject.

The components of the data transmission system are responsible for the transfer of the data from the subject. The sensor acquisition platform is equipped with node MCU which is used in transmission of sensor data. Sensors in the data acquisition part forms an IoT based architecture as each individual sensor's data can be accessed through the internet.

Data sensing is achieved by the sensors which are the foundation of vital signs monitoring system and responsible for the collection of data for measuring various physiological parameters. 
Data concentrator is used for concentrating the sensed and collected data to the android mobile app which is specifically designed for displaying the sensed output.

\subsection{IMPLEMENTATION}

This system mainly consists of temperature sensor, EMG sensor, the easily available and low-cost pulse sensor. The pulse sensor is noninvasively placed on the skin of the heart where the walls of the arteries are superficial, and the EMG sensor is placed on the arm muscles whereas the temperature sensor is placed near the tip of the finger. The readings are collected from the sensors and output is obtained through serial communication.

The $4.7 \mathrm{~K} \Omega$ resistor is connected between the signal and Vcc pin of DS18B20 sensor to ensure the correct output. The gnd pin of DS18B20 and pulse sensor is connected to the respective gnd pin of node MCU. The main purpose of using node MCU is to establish the Wi-Fi connection. The signal pin of the DS18B20 sensor is connected to D2 pin of the node MCU. The Vcc pins of DS18B20 and pulse sensor are connected separately in $3.3 \mathrm{~V}$ pin of node MCU.

The signal pin of the pulse sensor and DS18B20 sensor is connected to digital pins of the node MCU according to the requirement. The EMG sensor has 2 ground pins; one is connected to the node MCU and another one is connected to the batteries. The signal pin of the EMG sensor is connected to the A0 pin of the node MCU.

A controller node MCU is used to process the collected data and is an advanced Arduino Wi-Fi chip. The sensor data are obtained by serial communication with a Wi-Fi connection. When the Wi-Fi connection is established correctly without any interruption the output is obtained in serial monitor.

There are two modes of powering the circuit. i.e. USB and battery. The temperature sensor, pulse sensor, node MCU and raspberry pi are powered through the USB and the EMG sensor is powered by the battery.

The android app is created through which the output can be viewed. In android app, the data's can be viewed by connecting to same Wi-Fi which is given to node MCU. By connecting the same Wi-Fi, the output from the serial monitor is obtained in the android 
app. The android app is specially programmed to obtain the sensor data in real time. Using independent mobile application, user can login to IoT cloud and acquire vital signs monitoring data.

\section{RESULTS}

The normal ranges of the parameters observed clinically in this system are as follows:

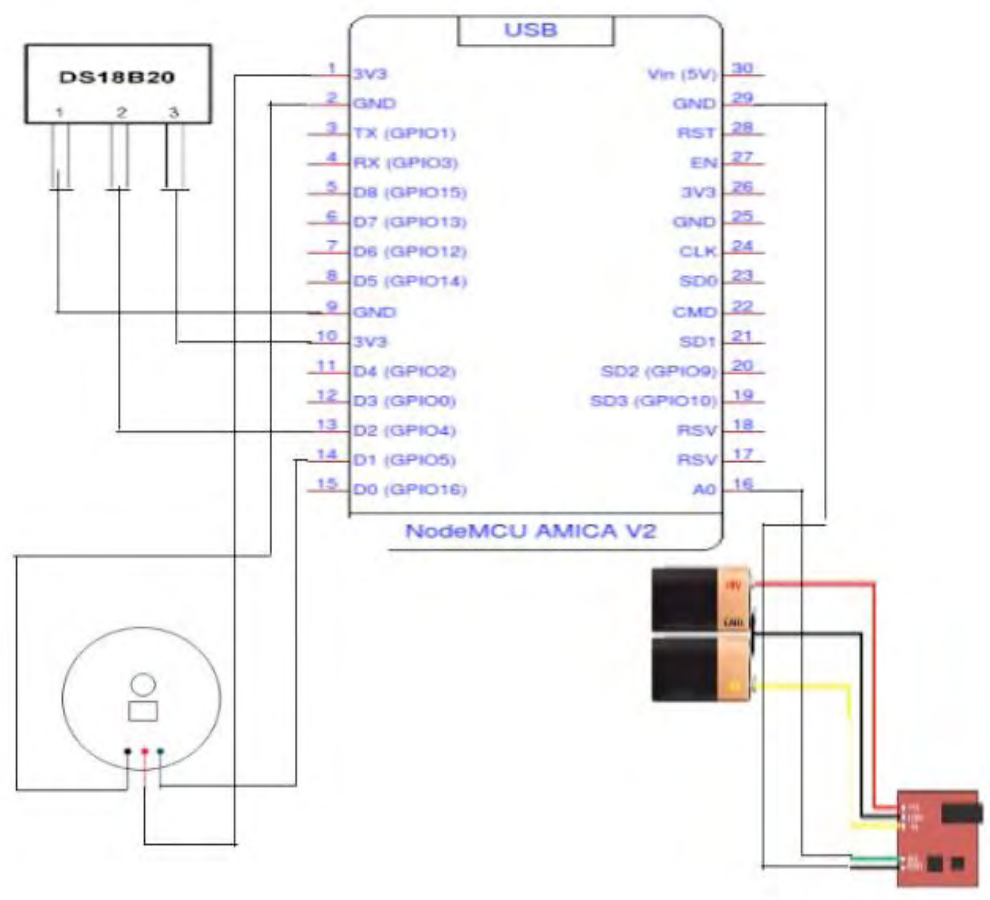

Figure 3. Interfacing of sensors with Node MCU.

Source: own elaboration.

Temperature sensor: 36.5 degree Celsius to 37.5 degree Celsius Pulse sensor: 60-80 bpm.

EMG: $50 \mu \mathrm{V}$ to $30 \mathrm{mV}$

Figure 3 represents the interfacing of different types of sensors with Node MCU in this system. The accuracy of our design is approximately more or less equal to clinical observation. The accuracy fluctuation may occur due to voltage supply and presence of noise during communication. 

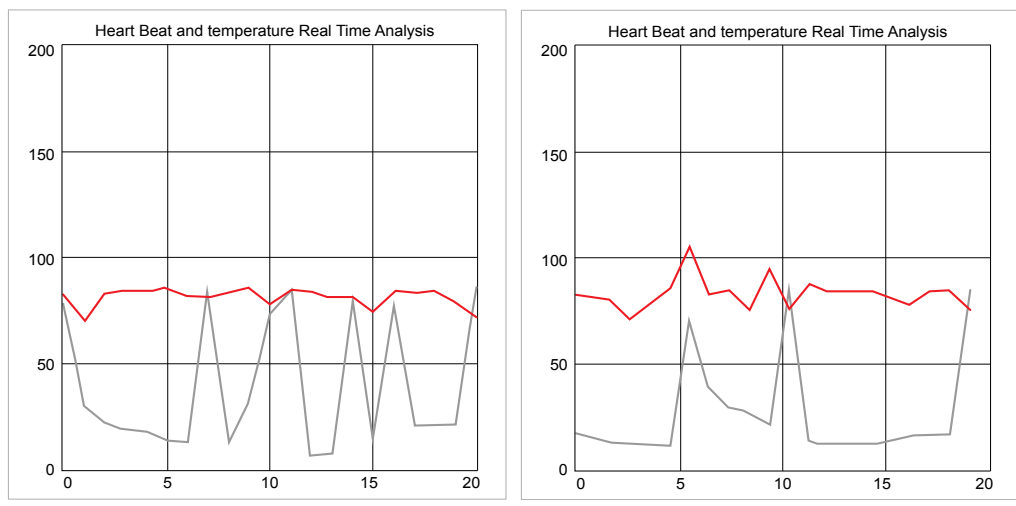

Figure 4. The graphs of sensor recorded from two persons using android app. In this figure grey line (-) represents temperature in Celsius and red line (-) represents pulse in bpm.

Source: own elaboration.

\section{CONCLUSIONS}

The existing healthcare monitoring system can provide vital parameters remotely using various technologies; the present work has multitudinous advantages for monitoring vital signs such as temperature, pulse rate and EMG of human health using easily available and low-cost sensors in a real-time manner. Thus, our system of health monitoring system can be used in case of emergency to be maintained and stored data regularly. It helps the medical professionals and patient to view their vital sign recording at the time of requirement. Thus, our design effectively measures even slight changes in the human body and transfers the medical output in the form of serial communication with confidentiality and integrity.

In future this device can be combined with cloud computing technology to store high number of data for longer period.

\section{ACKNOWLEDGMENT}

We would like to thank the management of Kalasalingam Academy of Research and Education for providing Summer Student Visiting Internship Program and We also express our sincere thanks and gratitude to Dr. SESHADHRI SRINIVASAN and Dr. B. SUBATHRA for their guidance and constant moral support throughout the completion of this project. 


\section{REFERENCES}

Castledine, G. (2006). The importance of measuring and recording vital signs correctly. British journal of nursing, 15(5), 285-285. https://doi.org/10.12968/ bjon.2006.15.5.20645

Gupta, S. D. M., Patchava, V. K., \& Menezes, V. (2015) Healthcare based on IoT using Raspberry Pi. In 2015 International Conference on Green Computing and Internet of Things (ICGCIo T). https://ieeexplore.ieee.org/document/7380571

Hassanalieragh, M., page, A., Soyata, T., Sharma, G., Aktas, M., Mateos, G., Kantarci, B., \& Andreescu, S. (2015). Health Monitoring and Management Using Internet-of-Things (IoT) Sensing with Cloud-based Processing: Opportunities and Challenges. In 2015 IEEE International Conference on service computing. https:// ieeexplore.ieee.org/document/7207365

Hong, W., Earnest, A., Sultana, P., Koh, Z., Shahidah, N., \& Ong, M. E. H. (2011). How accurate are Vital signs in predicting clinical outcomes in critically ill emergency department patients. European Fournal of Emergency Medicine, 20(1), 27-32. https:// journals.lww.com/euro-emergencymed/Fulltext/2013/02000/How_accurate_are_ vital_signs_in_predicting.6.aspx

Islam, S. M. R., Kwak, D., Kabir, M. H., Hossain, M., \& Kwak, K. (2015). The Internet of Things for Health Care: A Comprehensive Survey. IEEE Access, 3, 678708. https://doi.org/10.1109/ACGESS.2015.2437951

Malhi, K., Mukhopadhyay, S. G., Schnepper, J., Haefke, M., \& Ewald, H. (2012). A Zigbee-Based Wearable Physiological Parameters Monitoring System. IEEE Sensors fournal, 12(3), 423-430. https://doi.org/10.1109/JSEN.2010.2091719

Pantelopoulos, A., \& Bourbakis, N. G. (2010). A Survey on Wearable Sensor-Based Systems for Health Monitoring and Prognosis. IEEE Transactions on Systems, Man, and Cybernetics, Part C (Applications and Reviewes), 40(1), 1-12. https://doi.org/10.1109/ TSMCG.2009.2032660 
Patil, S., \& Pardeshi, S. (2018). Health Monitoring system using IoT. International Research Fournal of Engineering and Technology (IRFET), 5(4), 1679-1682. https://www.irjet.net/ archives/V5/i4/IRJET-V5I4375.pdf

Senthamilarasi, G., Rani, J. J., Vidhya, B., \& Aritha, H. (2018). A Smart Patient Health Monitoring System Using IoT. International fournal of Pure and Applied Mathematics, 119(16), 59-70. https:/ /www.acadpubl.eu/hub/2018-119-16/1/8.pdf

Yang, Z., Zhou, Q., Lei, L., \& Xiang, W. (2016). An IoT-cloud Based Wearable ECG Monitoring System for Smart Healthcare. Fournal of Medical Systems, 40, 286. https:/ / doi.org/10.1007/s10916-016-0644-9 